\title{
IN-STEP INFLATABLE ANTENNA EXPERIMENT
}

\author{
G. Veal \\ L'Garde, Inc. \\ Tustin, California \\ R. E. Freel and \\ Jet Propulsion Laboratory \\ California Institute of Technology \\ Pasadena, California
}

\author{
NASA/DOD \\ Flight Experiments \\ Technical Interchange Meeting \\ October 5-9, 1992/Monterey, California
}

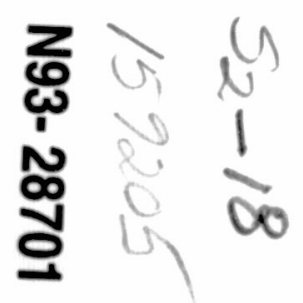




\section{IN-STEP INFLATABLE ANTENNA EXPERIMENT PRESENTATION CONTENTS}

- POTENTIAL SPACE ANTENNA APPLICATIONS

- EXPERIMENT OBJECTIVES

- EXPERIMENT TECHNICAL APPROACH

- EXPERIMENT SCENARIO

- SPARTAN SERVICES

- EXPERIMENT ORBITAL CONFIGURATION

- EXPERIMENT CANISTER STRUCTURE

- SURFACE MEASUREMENT SYSTEM CONFIGURATION

- ORBITAL FUNCTIONAL SEQUENCES

- SUMMARY 


\section{JPL}

\section{IN-STEP INFLATABLE ANTENNA EXPERIMENT POTENTIAL SPACE ANTENNA APPLICATIONS}

\begin{tabular}{|c|c|c|c|c|}
\hline \multicolumn{2}{|c|}{ APPLICATION } & $\begin{array}{c}\text { APERTURE } \\
\text { SIZE RANGE (M) }\end{array}$ & $\begin{array}{c}\text { RF } \\
\text { RANGE (GHz) }\end{array}$ & $\begin{array}{l}\text { INFLATABLE ANTENNA } \\
\text { APPLICATION POTENTIAL* }\end{array}$ \\
\hline \multicolumn{2}{|c|}{$\begin{array}{c}\text { MOBILE } \\
\text { COMMUNICATIONS }\end{array}$} & $10-20$ & $1.5^{\prime}$ & VERY HIGH \\
\hline \multicolumn{2}{|c|}{$\begin{array}{c}\text { MOBILE } \\
\text { COMMUNICATIONS }\end{array}$} & $4-8$ & $20-30$ & MODERATE \\
\hline \multicolumn{2}{|c|}{$\begin{array}{c}\text { EARTH } \\
\text { OBSERVATION } \\
\text { RADIOMETRY }\end{array}$} & $20-40$ & $6-60$ & MODERATE \\
\hline \multicolumn{2}{|c|}{$\begin{array}{l}\text { ACTIVE } \\
\text { MICROWAVE } \\
\text { SENSING }\end{array}$} & $\begin{array}{c}0.4 \times 2 \\
8 \\
4 \times 16\end{array}$ & $1-94$ & MODERATE \\
\hline OVLB & & $20-25$ & $0: 3-90$ & MODERATE \\
\hline $\begin{array}{l}\text { DOD SPA } \\
\text { BASED RA }\end{array}$ & & $20-30$ & $1.5-2.5$ & VERY HIGH \\
\hline CRITERIA & $\begin{array}{l}\text { - LOV } \\
\text { - LOV } \\
\text { - HIG }\end{array}$ & $\begin{array}{ll}\text { ST } & \bullet \text { HIGH } \\
\text { IGHT } & \bullet \text { PACK } \\
\text { LIABILITY } & \bullet \text { DIME }\end{array}$ & $\begin{array}{l}\text { CISION } \\
\text { NG EFFICIENCY } \\
\text { NAL STABILITY }\end{array}$ & - CONCEPT GROWTH POTENTIAL \\
\hline
\end{tabular}




\section{IN-STEP INFLATABLE ANTENNA EXPERIMENT EXPERIMENT OBJECTIVES}

- VALIDATE THE DEPLOYMENT OF A 14 METER INFLATABLE PARABOLIC REFLECTOR STRUCTURE IN A ZERO GRAVITY ENVIRONMENT

- INFLATABLE ELEMENT DEPLOYMENT SEQÚENCE

- DEPLOYMENT RATES

- MEASURE THE REFLECTOR SURFACE ACCURACY UNDER ORBITAL MECHANICAL AND THERMAL LOADING CONDITIONS

- FIVE SUN ANGLES

- THREE REFLECTOR/CANOPY PRESSURES

- INVESTIGATE REFLECTOR STRUCTURE DAMPING CHARACTERISTICS UNDER ORBITAL OPERATIONAL CONDITIONS

- EXCITE FUNDAMENTAL NODES

- MEASURE AMPLITUde DECAY 


\section{IN-STEP INFLATABLE ANTENNA EXPERIMENT TECHNICAL APPROACH}

- SPARTAN RECOVERABLE SPACECRAFT AS EXPERIMENT CARRIER

- MOUNTING PLATFORM

- POWER

- ATtTITUDE CONTROL

- DATA RECORDING

- REFLECTOR STRUCTURE BASED ON SOLAR CONCENTRATOR TECHNOLOGY

- CONFIGURATION IDENTICAL

- CONCEPT DEVELOPMENT OF STRUCTURES UP TO 9 meters

- INFLATION SYSTEM BASED ON FLIGHT PROVEN DESIGNS

- ANTENNA DEPLOYMENT MONITORED WITH VIDEO CAMERAS

- WIDE AND NARROW ANGLE CAMERAS

- FLIGHT QUALIFIED CAMERAS

- SURFACE PRECISION MEASURED WITH DIGITAL IMAGING RADIOMETER

- PROVEN CONCEPT

- GROUND BASED CALIBRATION

- STRUCTURAL DAMPING DERIVED FROM MOTION DECAY PLOTS

- STRUCTURAL EXCITATION PROVIDED BY SPARTAN CONTROL SYSTEM

- MOTION DECAY MEASURED WITH TRANSDUCERS 


\section{JPL IN-STEP INFLATABLE ANTENNA EXPERIMENT}

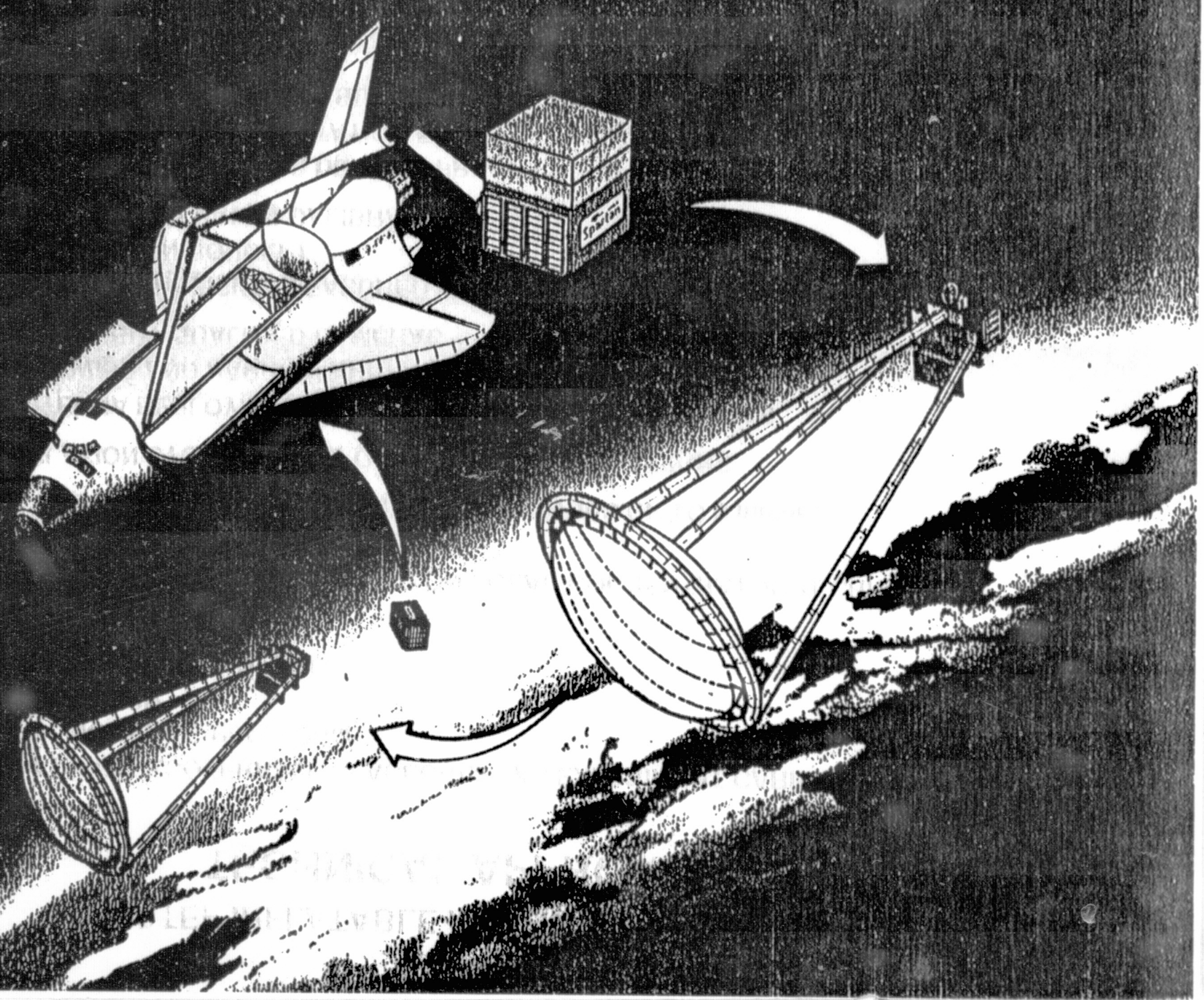




\section{IN-STEP INFLATABLE ANTENNA EXPERIMENT SPARTAN SERVICES}

- EXPERIMENT CARRIER

- EXPERIMENT INTERFACE WITH STS

- thermal control (PRIOR to DePloyment)

- ATTITUDE CONTROL.

- electrical POWER

- DATA RECORDING

- ORBIT POSITION AND ATTITUDE VS. TIME DATA

- EXPERIMENT INITIATION SIGNAL (TWO FAULT TOLERANT)

- EXPERIMENT/SPARTAN SEPARATION SYSTEM 


\section{JPL EXPERIMENT ORBITAL CONFIGURATION}

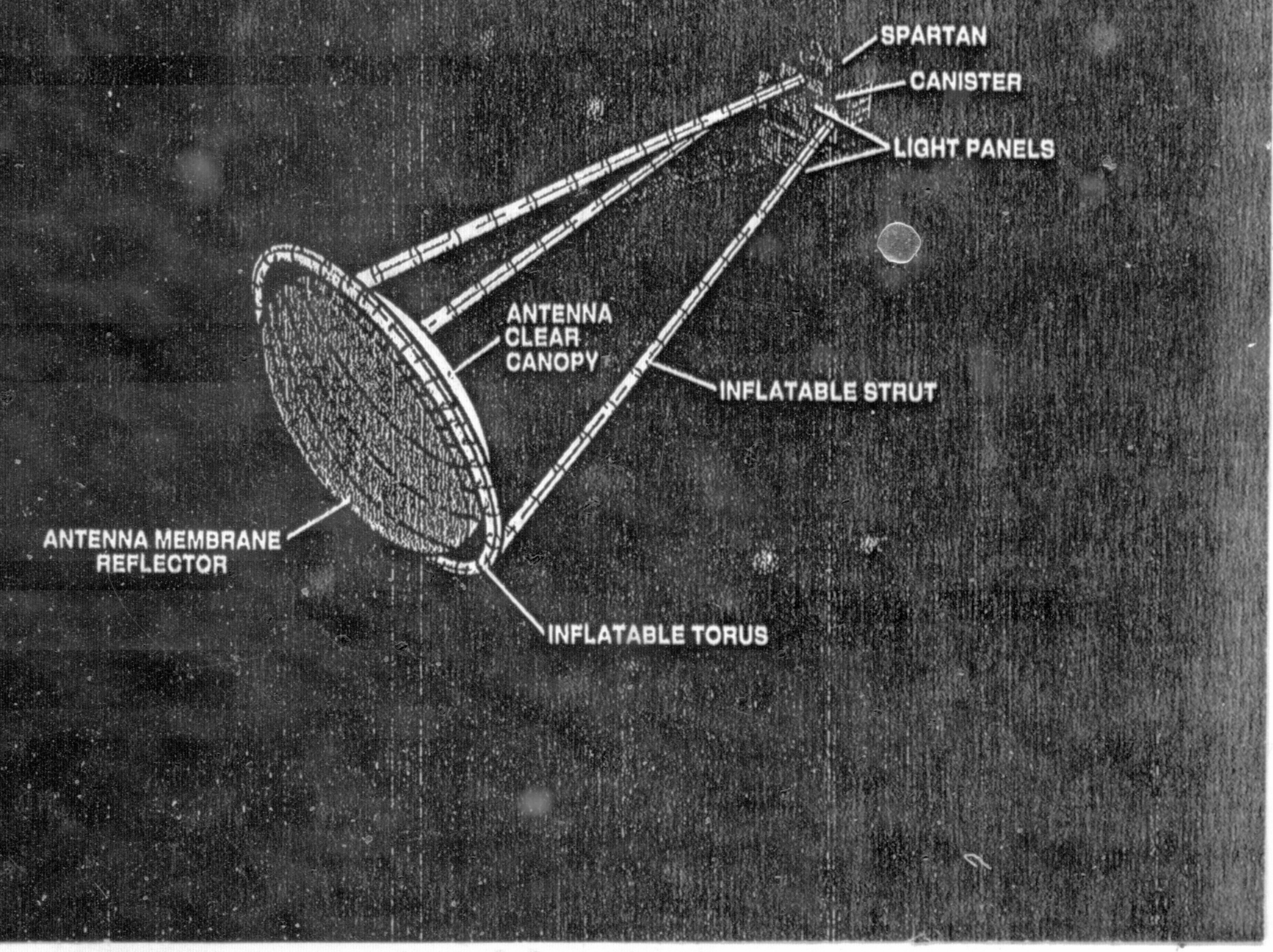




\section{IN-STEP INFLATABLE ANTENNA EXPERIMENT CANISTER AND SPARTAN}

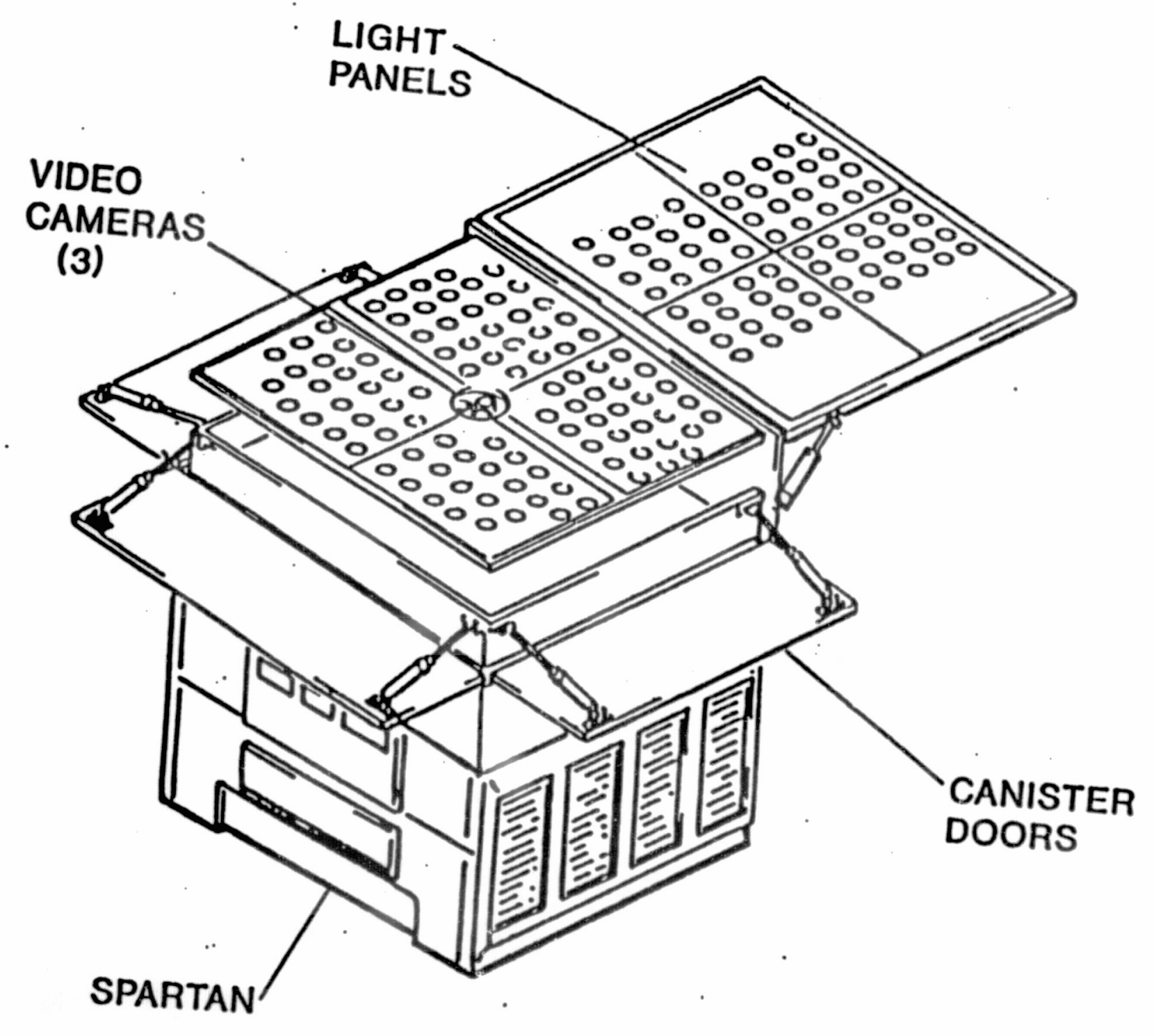




\section{IN-STEP INFLATABLE ANTENNA EXPERIMENT SURFACE MEASUREMENT SYSTEM CONFIGURATION}

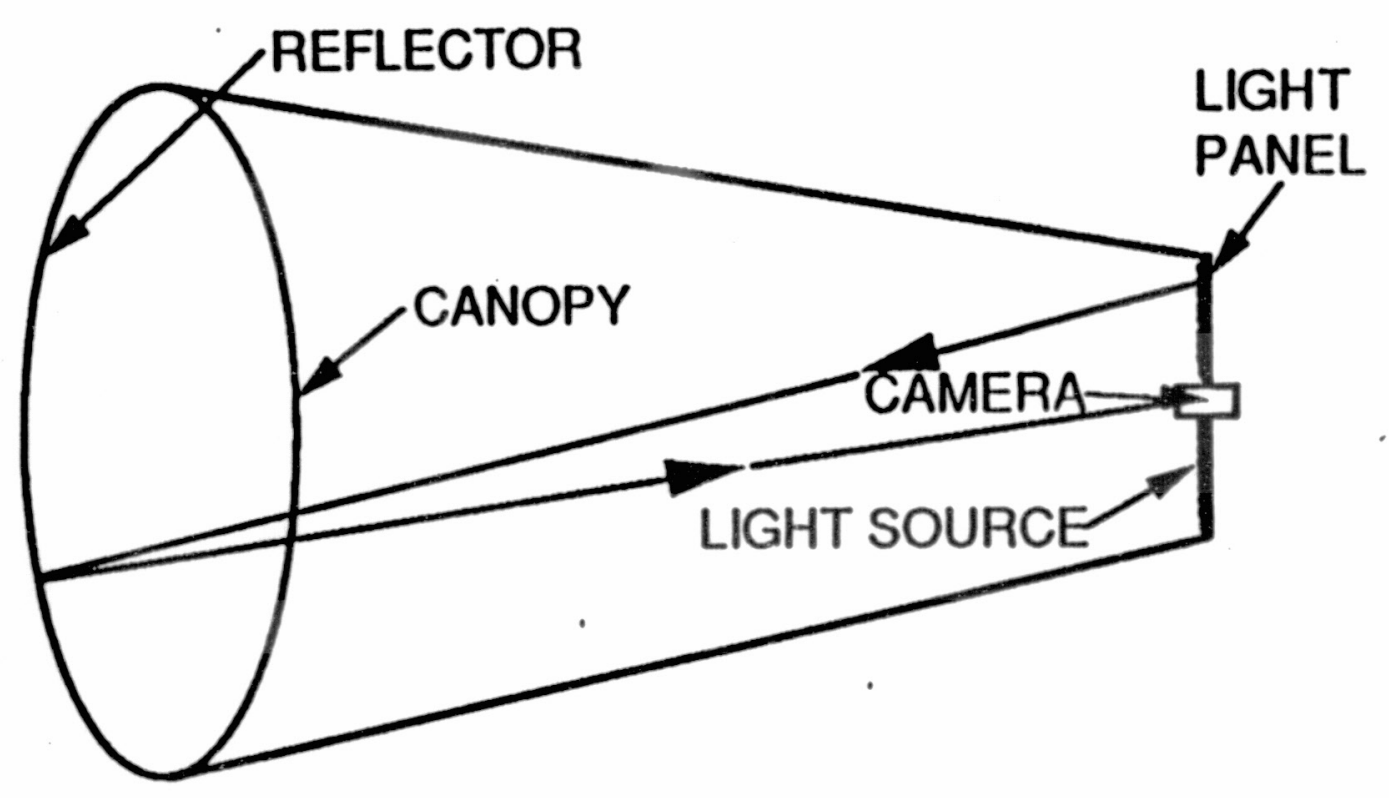




\title{
. IN-STEP INFLATABLE ANTENNA EXPERIMENT ORBITAL FUNCTIONAL SEQUENCES
}

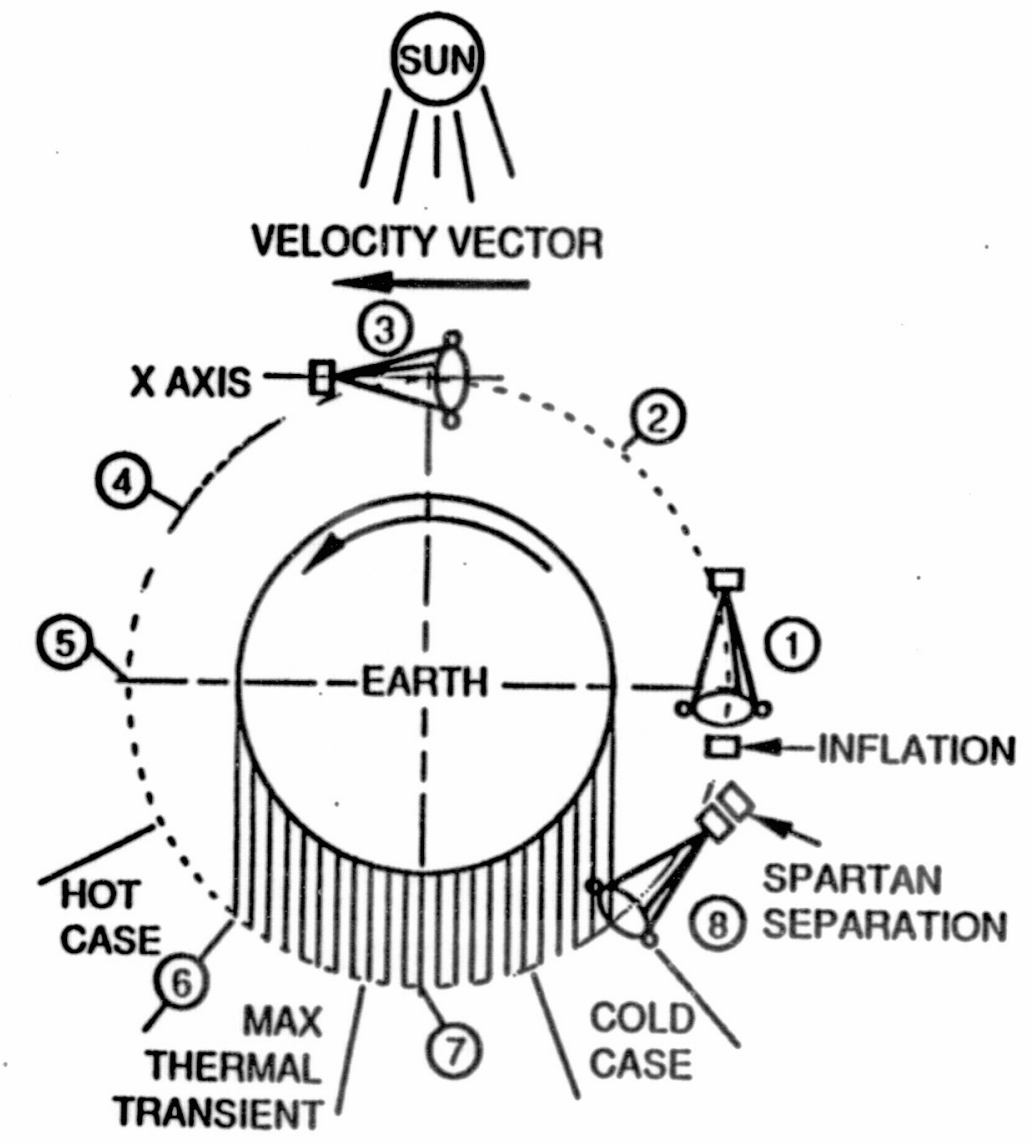

\author{
ORBITAL FUNCTIONS \\ - EXPERIMENT INITIATION COMMAND FROM SPARTAN \\ - CANISTER door DEPLOYMENT \\ - REFLECTOR STRUCTURE DEPLOYMENT BY INFLATION \\ - VIDEO COVERAGE OF REFLECTOR DEPLOYMENT \\ - SURFACE MEASUREMENT AS FUNCTION OF \\ - SUN ANGLE: ORBITAL POSITIONS 1-5 \\ - CANOPY PRESSURE: ORBITAL POSITION 6-8 \\ - ANTENNA STRUCTURE EXCITATION PROVIDED BY \\ SPARTAN \\ - MEASUREMENT OF STRUCTURE AMPLITUdE \\ DECAY \\ - ANTENNA/SPARTAN SEPARATION
}




\section{IN-STEP INFLATABLE ANTENNA EXPERIMENT SUMMARY}

- PROgram STAtus

- preliminary design Complete

- CONCEPTUAL DESIGN REVIEW ACCOMPLISHED

- CARRIER INTERFACE ESTABLISHED

- PHASE 0 SAFETY REVIEW IN PROCESS

- PHASE C/D PROGRAM PLANNING COMPLETE

- EXPERIMENT RESULTS

- DEPLOYMENT RELIABILITY VALIDATED BY EXPERIMENT

- LOW WEIGHT AND VOLUME DEMONSTRATED BY FABRICATION OF LARGE SIZE STRUCTURE

- LOW STRUCTURE COST VERIFIED BY LOW COST EXPERIMENT

- USER POTENTIAL DETERMINED BY RESULTS OF SURFACE MEASUREMENT

- ESTABlish CONCEPT TECHNOLOgY DATA BASE

- PROJECTIONS OF PERFORMANCE FOR DIFFERENT APPLICATIONS 